\title{
CONCEPÇÃO DE UM SOFTWARE PARA UTI-ADULTO DO HCU-UFU
}

\author{
H. B. Bueno*, I. W. Oliveira*, E. M. Carvalho*, P. A. Borges* e S. T. Milagre* \\ *Universidade Federal de Uberlândia, Faculdade de Engenharia Elétrica Uberlândia, Brasil \\ e-mail: hudsons.bruno@hotmail.com
}

Resumo: O setor de Unidade de Terapia Intensiva (UTI) do Hospital de Clínicas de Uberlândia da Universidade Federal de Uberlândia (HCU-UFU) é um setor muito importante para o tratamento de pacientes em estados críticos que necessitam de um monitoramento contínuo de seus sinais vitais. Porém, com o processo de admissão de paciente na UTI sendo em documento físico, muito tempo dos profissionais é redirecionado para processos burocráticos em si, e não para com a assistência à saúde. O objetivo desse projeto foi a concepção de um software para automatizar esse processo. Assim o software foi desenvolvido no setor de Tecnologia de Informação do HCU-UFU. Os resultados obtidos foram as interfaces de controle de pacientes da UTI-Adulto e a otimização da geração de relatórios.

Palavras-chave: HCU-UFU, UTI-Adulto, software, admissão de paciente.

\begin{abstract}
The ICU sector of Uberlândia Clinical Hospital of Federal University of Uberlândia (HCUUFU) is a very important sector for the treatment of patients in critical conditions that require continuous monitoring of vital signs. However, with patient intake process in the ICU being in physical document, the time of the professionals is redirected to bureaucratic processes themselves, health care. The aim of this project was to design a software to automate this process. Therefore, the software was developed in the Information Technology sector HCU-UFU. The results obtained were the interfaces that control patients of the adult ICU and optimization of the generation of reports. The software has not yet been implemented in the sector in question, because there is only the development of a screen left.
\end{abstract}

Keywords: HCU-UFU, adult ICU, software, patient admission.

\section{Introdução}

Os serviços em Unidades de Terapia Intensiva têm por objetivo prestar atendimento a pacientes graves e de risco, os mesmos necessitam de assistência médica com supervisão 24 horas, além de equipamentos e recursos humanos especializados para cada caso [1].

Projetar dados dos pacientes em planilha não é uma demanda simples de ser realizada, e muitas vezes, algumas delas são executadas de forma manual, fazendo parte da rotina dos muitos profissionais da área e no caso dos setores de UTIs não são diferentes, com este procedimento rotineiro sendo executado na UTI do Hospital de Clinicas de Uberlândia da Universidade Federal de Uberlândia (HCU-UFU), hoje, se faz necessário de um sistema integrado para automatizar os processos organizando documentos e dados.

$\mathrm{Na}$ UTI do HCU-UFU, por exemplo, foi desenvolvido um software, em Access, por um antigo profissional que trabalhava no setor. O software apresenta alguns problemas, tais como atualmente não atende as necessidades que o setor exige, não é compatível com o sistema operacional usado pelo hospital, não é conectado com o banco de dados do hospital necessitando de inserção manual de alguns dados do paciente e, além disso, o sistema controle de dados do paciente é completamente manual por meio de um caderno de anotações, o que não é uma solução eficaz para o setor, visto que o serviço de secretaria responsável deve repassar estes dados ao hospital, com isso perde-se muito tempo que útil neste processo.

Neste contexto, com o objetivo de auxiliar na gestão de admissão de pacientes na UTI-Adulto do HCU-UFU com a finalidade de otimizar o tempo dos profissionais de saúde dispendido nessa atividade foi proposto um software de controle do fluxo de pacientes ligado diretamente ao banco de dados do hospital.

\section{Materiais e métodos}

A metodologia utilizada foi descritivo-ativa, com técnica ativa em campo, análise documental e desenvolvimento de um sistema computacional, com vistas ao gerenciamento dos leitos presentes na UTIAdulto do HCU-UFU segundo simples controle de admissão dos pacientes.

O projeto foi uma demanda existente e explicitada pela profissional responsável pelo setor a fim de integrar o sistema de admissão dos pacientes no sistema computacional de gestão do hospital. Assim, para o desenvolvimento do mesmo, foi necessário projetar o software dentro dos padrões específicos utilizados pelo Centro de Tecnologia da Informação (CTI-UFU) do HCU-UFU. Portanto, o sistema foi planejado de forma a atender os requisitos técnicos e funcionais de todos os setores envolvidos.

Para fins de desenvolvimento do software, foi adotado o método do "Modelo Cascata de Produção" [2] que consiste no fato de que cada etapa deve ser 
finalizada antes de iniciar uma nova etapa do projeto, onde as mesmas são geralmente divididas em Requerimento, Projeto, Implementação, Verificação e Manutenção (Figura 1).

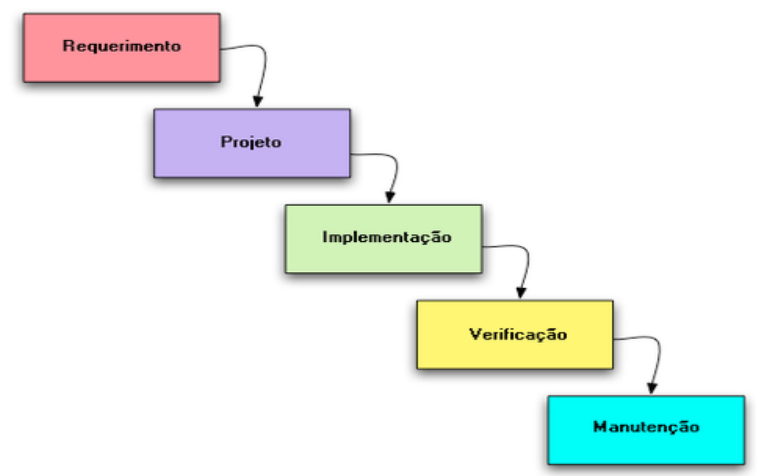

Figure 1: Modelo Cascata de Produção [2].

O CTI-UFU utiliza de rede interna e sistemas próprios, além de padronização das ferramentas utilizadas tanto para desenvolvimento quanto para manutenção e uso das tecnologias da informação presentes na UFU. Portanto toda a atividade de desenvolvimento do projeto se passou no CTI-UFU, já que era necessário se conectar à rede para acessar o banco de dados e para comitar o projeto no sistema do hospital.

Além de estar conectado à rede do HCU-UFU e devido à falta de uma máquina disponível para o desenvolvimento do projeto foi necessário a configuração dos computadores pessoais dos componentes do grupo do projeto, fase que durou cerca de dois meses devido à complexidade do processo e a necessidade de se ter um técnico disponível para realizar tal configuração.

O padrão de desenvolvimento do CTI-UFU utiliza para interfaces a linguagem HTML [3], para ações a linguagem JAVA [4] e para armazenamento de dados o script DB2 [5]. Assim o desenvolvimento em JAVA é realizado por meio de um software baseado em ECLIPSE, o JBOSS STUDIO [6], o banco de dados é programado em linguagem script DB2 e gerenciado por mio do AQUA DATA STUDIO [7] e as telas para interface em HTML, sendo que JBOSS STUDIO é responsável por realizar a comunicação entre todos os softwares utilizados no projeto. Além disso os relatórios também são incluídos nessa interface de gerenciamento e são incialmente desenvolvidos no TIBCO JASPERSOFT STUDIO [8].

\section{Resultados}

Obteve-se com desenvolvimento do projeto, grande parte da interface de gerenciamento dos leitos da UTIAdulto. Para efeito de comparação, tem-se na Figura 2 como era a disposição da interface do software existente no setor da UTI-Adulto, desenvolvido em Access. A Figura 2 mostra parte da tela de gerenciamento de leitos, evidenciando os pacientes da Unidade II. Vale ressaltar que todas as informações dos pacientes existem no banco de dados do hospital, mas são digitadas à mão para alimentar o software do setor.

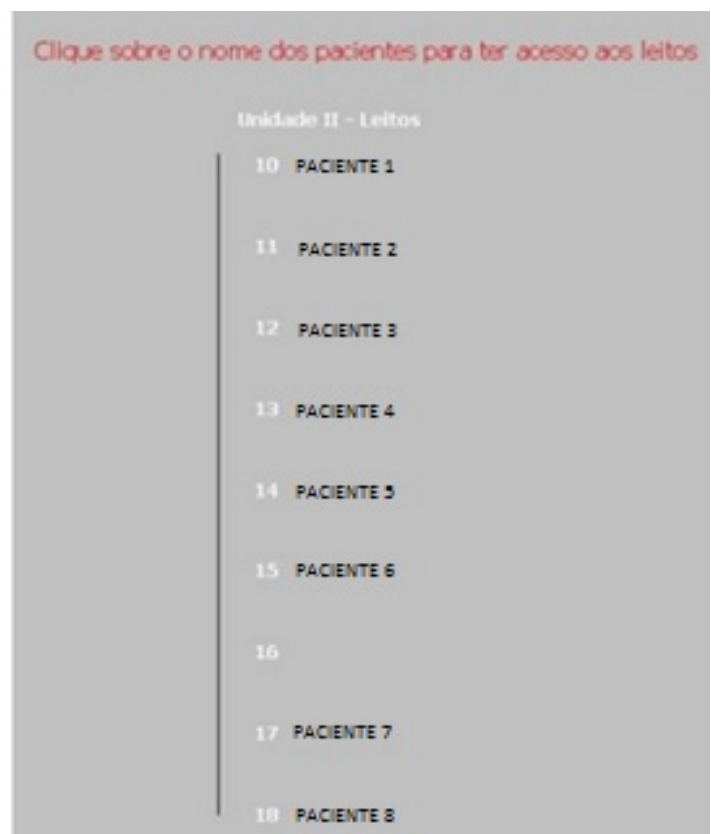

Figura 2: Tela de gerenciamento de leitos do software em Access.

Já as telas de interface desenvolvidas de acordo com o padrão utilizado pelo setor da Tecnologia da Informação estão representadas na Figura 3 e Figura 4. A primeira é a tela análoga a Figura 2, mas com a nova configuração e conectada ao banco de dados para o preenchimento automático dos dados.

\begin{tabular}{|c|c|}
\hline \multicolumn{2}{|r|}{ Unidde III- Létos } \\
\hline Lath & Nome \\
\hline 10 & PACIENTE 1 \\
\hline 11 & PACIENTE 2 \\
\hline 12 & PACIENTE 3 \\
\hline 13 & PACIENTE 4 \\
\hline 14 & PACIENTE 5 \\
\hline 15 & PACIENTE 6 \\
\hline 16 & PACIENTE 7 \\
\hline 17 & PACIENTE 8 \\
\hline 18 & PACIENTE 9 \\
\hline
\end{tabular}

Figura 3: Tela de gerenciamento de leitos do software desenvolvido.

Já a Figura 4 mostra o dashboard que contém as ações que podem ser realizadas pelo usuário do sistema, como por exemplo, cadastrar uma adenda de alta cirúrgica e um pré-operatório, consultar tais cadastros e visualizar o painel de internação.

Por fim, para cada leito, ou seja, para cada paciente, alguns relatórios são gerados quando necessários, como por exemplo uma solicitação de gastrostomia, um termo 
de compromisso para gastrostomia endoscópica, tabela com exames diários, entre outros.

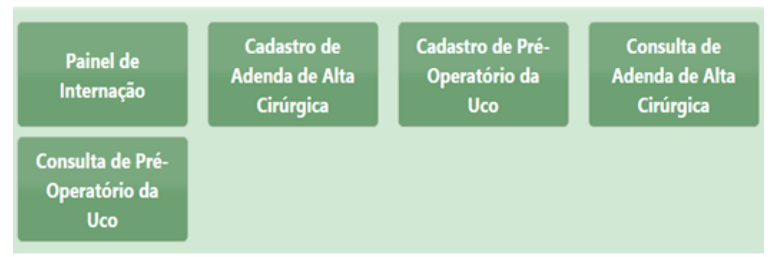

Figura 4: Dashboard com as ações que podem ser realizadas no software desenvolvido.

Esses relatórios foram desenvolvidos e quando solicitados a partir de um paciente, seus dados são preenchidos automaticamente no relatório.

\section{Discussão}

Primeiramente, grande parte do tempo de projeto foi dedicado para a formatação dos computadores a serem utilizados no desenvolvimento da interface e na configuração de detalhes técnicos.

Como dificuldades encontradas, tem-se a compreensão dos códigos do sistema do hospital e do banco de dados que não apresentam documentação em sua grande parte.

Além disso, a comunicação com um banco de dados tão grande se tornou um desafio a ser enfrentado e a criação de relatórios dinâmicos é um ponto bastante complexo, pois deve-se fazer a comunicação entre o relatório desenvolvido no TIBCO JASPERSOFT, o banco de dados e a aplicação em JAVA.

Um dos grandes empecilhos para a implantação efetivado software na UTI-Adulto do HCU-UFU foi o problema relacionado a configuração dos computadores pessoais para se conectar à rede do Hospital, pois a cada conexão os computadores se desconfiguravam e as versões do projeto entre os computadores pessoais e o sistema do CTI-UFU se desatualizavam.

Por fim, a transposição do software do Access para os padrões da TI apresentou dificuldades no início, mas com o tempo e com a familiarização do ambiente e das ferramentas de trabalho, o desenvolvimento do projeto foi se tornando mais fácil.

Fora esses detalhes, o desenvolvimento das telas e dos relatórios em si é um processo rápido e simples.

\section{Conclusão}

Esse projeto procurou automatizar alguns documentos e organizar de forma digital o controle de pacientes da UTI-Adulto, de modo a facilitar busca de dados, criação de indicadores e agilizar o processo em si.

Como grande parte da equipe de projeto tinha conhecimentos básicos das linguagens de programação utilizadas no software assim como pouco ou nenhum conhecimento das ferramentas utilizadas no setor de Tecnologia da Informação do HCU-UFU, como Aqua
Data Studio e o JBoss, foi um grande aprendizado técnico o desenvolvimento desse projeto.

Além disso, percebe-se que há uma grande oportunidade de atuação na área de Tecnologia da Informação, para auxiliar o fluxo de dados que ocorre dentro do hospital. Há uma grande demanda de projetos a serem realizados pelo setor, mas pouco tempo para colocar todos em execução. Assim, estudantes dispostos a aprender sobre a área e com vontade de realizar um projeto que fará a diferença no hospital são bem-vindos.

Por fim, espera-se que o software de gestão da UTIAdulto desenvolvido neste projeto seja capaz de auxiliar, agilizar e facilitar o serviço tão complexo e importante que é realizado neste setor.

\section{Agradecimentos}

Agradecemos a Professora Selma Terezinha Milagre pelo incentivo, motivação e orientação deste trabalho, a toda a equipe do Centro de Tecnologia da Informação do Hospital das Clínicas de Uberlândia que forneceu toda a infraestrutura para a concepção desse projeto e a Doutora Liliane pela oportunidade oferecida a nós estudantes do curso de Engenharia Biomédica da Universidade Federal de Uberlândia.

\section{Referências}

[1] GRIMES, S. L. The Future of Clinical Engineering: The Challenge of Change. IEEE Engineering in Medicine and Biology Magazine. Março, 2003. 9199.

[2] PRESSMAN ROGER S. Engenharia de Software: Uma Abordagem Profissional. [S.1.]: [s.n.], 2011.

[3] FREEMAN, E. Use a Cabeça! Programação em HTML5. 1. ed. [S.1.]: Alta Books, 2014. ISBN 97885-7608-845-5.

[4] SIERRA, K.; BATES, B. Use a Cabeça JAVA. 1. ed. [S.1.]: Alta Books, 2005. ISBN 978-85-7608-173-9.

[5] FERRARI SILVIO. Introdução ao IBM DB2 Universal Database. 1. ed. [S.1.]: IBM, 2011. ISBN 9788573936711.

[6] JBOSS STUDIO. Release Notes, 2016. Disponivel em: <www.access.redhat.com/documentation/enUS/Red_Hat_JBoss_Developer_Studio/9.1/html/9.1. 0_Release_Notes/index.html $>$. Acesso em: 27 maio 2016 .

[7] AQUAFOLD. Aqua Data Studio, 2016. Disponível em: $<$ www.aquafold.com/aquadatastudio.html $>$ Acesso em: 28 maio 2016.

[8] JAS PERSOFT C OMMUNITY. TIBCO JasperReports Server v6.0.1 Release Note, 2016. D i s p o n í ve 1 e $\mathrm{m}$ $<$ www.community.jaspersoft.com/wiki/tibcojasperreports-server-v601-release-notes>. Acesso: 03 abr. 2016. 